\title{
Möglichkeiten und Grenzen der Analyse von Holzmärkten
}

\author{
Roland Olschewski \\ Oliver Thees
}

Eidgenössische Forschungsanstalt für Wald, Schnee und Landschaft (CH)*

Eidgenössische Forschungsanstalt für Wald, Schnee und Landschaft (CH)

\section{Chances and limits of the analysis of wood markets}

\begin{abstract}
Recent approaches of behavioural economics and agent-based modeling can enhance knowledge about market processes and results and widen the focus for the assessment of future market developments by emphasising the individual behaviour of market participants and scenario techniques. In this article we resume possible contributions of the particular approaches to better describe, explain and forecast real market developments. The exposition is based on state-of-the-art knowledge and reflects insights gained during the 8th Forest Economic Seminar in autumn 2011, where researchers and practitioners presented their findings.
\end{abstract}

Keywords: econometrics, micro economics, behavioural economics, agent-based modeling, market analysis doi: $10.3188 /$ szf.2012.0396

*Zürcherstrasse 111, CH-8903 Birmensdorf, E-Mail roland.olschewski@wsl.ch

$\mathrm{D}$ er Forstsektor hat ein grosses Potenzial zur Bereitstellung nachwachsender Rohstoffe und kann damit wesentlich zur nachhaltigen Entwicklung in der Schweiz beitragen. Tatsächlich ist die Biomasseproduktion insgesamt aber begrenzt, und unterschiedliche Akteure konkurrieren um die Verwendung des nachwachsenden Holzes. Dabei ist zu beachten, dass die physisch vorhandenen Holzvorräte in Schweizer Wäldern nicht gleichzusetzen sind mit der tatsächlich auf Märkten verfügbaren Holzmenge. Es gilt daher, realistische Informationen über das zukünftig zu erwartende Holzaufkommen zu gewinnen. In diesem Zusammenhang sind verschiedene Marktbesonderheiten festzustellen. Eine kürzlich erschienene Studie hat gezeigt, dass viele Schweizer Waldeigentümer nur in geringem Ausmass auf Preisänderungen reagieren (Pauli et al 2010). Ein Grund dafür kann in dem sogenannten «Geringfügigkeitsproblem» gesehen werden. Viele Waldeigentümer verfügen nur über relativ kleine Parzellen, was zu vergleichsweise hohen Ernte- und Transaktionskosten führt und den Markteintritt erschwert. Zusätzlich haben natürliche Risiken, zum Beispiel Sturmereignisse oder Insektenkalamitäten, Einfluss auf das Verhalten von Waldbesitzern und die mit der Bewirtschaftung betrauten Förster, was dazu führen kann, dass diese eher kurzfristige Verträge abschliessen, statt sich langfristig zu binden. Schliesslich haben auch die
Eigentümerstruktur und gesetzliche Regelungen, etwa bei der Schutzwaldbewirtschaftung, Auswirkungen auf das Marktangebot. All diese Aspekte können dazu führen, dass tatsächlich weniger Holz für die Volkswirtschaft verfügbar ist als nachhaltig zuwächst. Um die Entwicklung des zukünftigen Holzaufkommens besser abschätzen zu können, ist es daher wichtig, zu erfahren, wie sich Holzanbieter und -nachfrager unter bestimmten Bedingungen verhalten und weshalb.

Zur Abschätzung von Mengen- und Preisentwicklungen auf Holzmärkten stehen verschiedene Analysemethoden zur Verfügung. Dabei werden sowohl ökonometrische Schätzungen auf Basis von Zeitreihenanalysen durchgeführt als auch Experteninterviews genutzt, um Erkenntnisse über das Angebots- und Nachfrageverhalten der Akteure auf Holzmärkten zu gewinnen (Bergen et al 2002, Michels 1991, Michels \& Steinmeyer 1992, Moog 1992, Steinmeyer 1992a, b, Thoroe et al 1998, Schwarzbauer \& Stern 2010; Abbildung 1).

Darüber hinaus können neuere Ansätze der Verhaltensökonomie und der agentenbasierten Modellierung (ABM) das Verständnis des Marktgeschehens, etwa durch Fokussierung auf das individuelle Verhalten der Akteure und Szenarioanalysen, erweitern und damit einen Beitrag zur Beurteilung zukünftiger Entwicklungen leisten (Armstrong \& Porter 2007, Olschewski et al 2009). Im Folgenden wird 


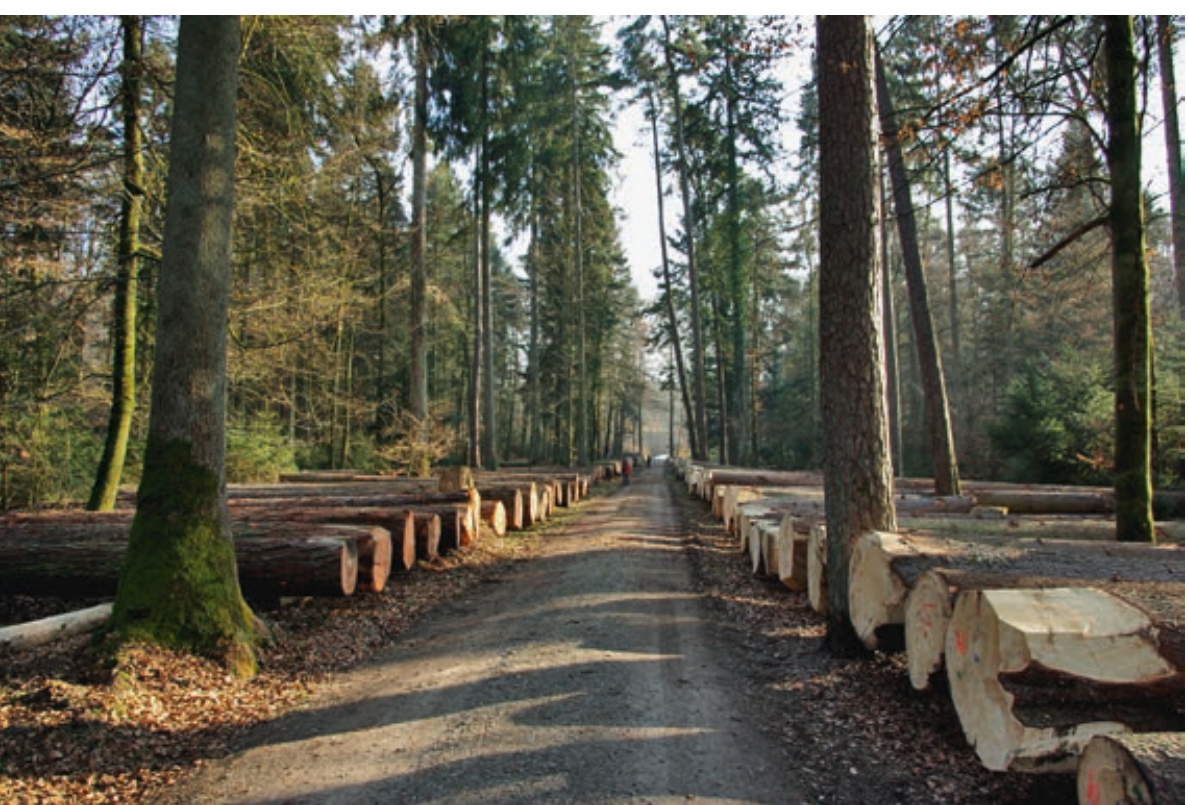

Abb 1 Der Wertholzmarkt ist einer von verschiedenen Holzmärkten. Hier ist die Submission das übliche Verkaufsverfahren. Foto: Anton Bürgi (WSL)

untersucht, welchen Beitrag die beschriebenen Ansätze leisten können, um die Realität der Märkte zu beschreiben, zu erklären und vorherzusagen. Die Ausführungen basieren auf dem aktuellen Stand des Wissens und reflektieren die Erkenntnisse des 8. Waldökonomischen Seminars, das im Herbst 2011 stattfand. Dort wurden zum einen die Grundlagen der ökonomischen und empirischen Holzmarktanalyse vorgestellt; zum anderen wurde eine Einführung in die Verhaltensökonomie und in die agentenbasierte Modellierung gegeben. Schliesslich wurden Akteure auf der Angebots- und Nachfrageseite von Holzmärkten gebeten, ihre praktischen Einsichten in die Funktionsweise von Märkten zu präsentieren und zur Diskussion zu stellen.

\section{Grundlagen der ökonomischen Holz- marktanalyse}

Die Marktanalyse dient dem Ziel, beobachtete Marktergebnisse zu erklären. Um den Rahmen für die Analyse zu bestimmen, ist eine Marktabgrenzung in sachlicher, räumlicher und zeitlicher Hinsicht vorzunehmen (Bergen et al 2002). Im Folgeschritt wird die Marktform bestimmt. Abhängig von der Anzahl der Teilnehmer auf beiden Marktseiten liegt entweder ein Konkurrenzmarkt (Polypol), ein Oligopol, ein Monopol oder eine monopolistische Konkurrenz vor (Mankiw \& Taylor 2008). Dabei ist weiterhin zwischen einem vollkommenen und einem unvollkommenen Markt zu unterscheiden, je nachdem, ob das gehandelte Gut immer die gleichen Eigenschaften aufweist (Homogenität) oder verschiedenartig ist (Heterogenität). Aus Sicht der Nachfrager und Anbieter besteht gegebenenfalls die Möglichkeit, Substitute mit in die Kauf- beziehungsweise Verkaufsentscheidung einzu- beziehen. In diesem Fall ist ein möglicher Einfluss auf das Marktergebnis mit zu berücksichtigen.

Bei der Betrachtung von Holzmärkten sind zusätzlich forstspezifische Besonderheiten zu beachten, die das Verhalten der Marktteilnehmer insbesondere auf der Angebotsseite beeinflussen. Dazu gehören unter anderem 1) die Koppelproduktion verschiedener Sortimente, 2) Pflegeeingriffe, die unabhängig vom Marktgeschehen anfallen, 3) die Beachtung der nachhaltigen Holzproduktion, 4) die Festlegung des Erntezeitpunkts innerhalb des Jahres abhängig von ökologischen und ökonomischen Faktoren und 5) die Unsicherheiten bezüglich der Auswirkungen des Klimawandels auf den Wald.

Darüber hinaus können unterschiedliche Verhaltenshypothesen bezüglich der Ziele der Marktteilnehmer aufgestellt werden. Generell können als Ziele die Maximierung des Gewinns, die Deckung der Kosten sowie die Produktion einer bestimmten (konstanten) Erntemenge pro Jahr angestrebt werden (Bergen et al 2002). Für die jeweilige Zielerreichung ist es wichtig, das zukünftige Handeln auf Basis von Erwartungen zu planen. Diese Erwartungsbildung kann beispielsweise extrapolativ erfolgen, d.h. durch Fortschreibung der Vergangenheitsinformationen, ohne dass ein Lernen zugelassen wird. Eine adaptive Erwartungsbildung ist ebenfalls auf die Vergangenheit bezogen, die Akteure sind aber in der Lage, aus Fehlern zu lernen. Die rationale Erwartungsbildung unterstellt einen Lernprozess unter Verwendung von Vergangenheits- und Gegenwartsinformationen (Miller 2003).

In dem von Volker Bergen entwickelten Rohholzmarktmodell wird unterstellt, dass die Marktakteure rationale Erwartungen haben. Er kommt in seinem Beitrag zu dem Schluss, dass sich der Rohholzmarkt als ein Markt mit normal verlaufenden Angebots- und Nachfragekurven beschreiben lässt (Bergen 2012, dieses Heft). Die empirische Marktanalyse liefert dabei brauchbare Prognosen allerdings nur auf kurze Sicht.

\section{Verhaltensökonomische Grundlagen der Marktanalyse}

Ökonometrische Modelle wie das zuvor beschriebene Holzmarktmodell basieren häufig auf der Annahme rational handelnder Akteure. Diese bildet quasi einen Extrempunkt einer grossen Spannbreite beobachtbaren Verhaltens, welche vom nutzenmaximierenden homo oeconomicus mit konstanten Präferenzen bis hin zu einem total auf Zufall basierenden Verhalten reichen kann (Shogren et al 2010). Die einzige grössere Abweichung von der Rationalitätsannahme war für lange Zeit die Berücksichtigung von Risikoscheu. Kahneman \& Tversky (1979) verwendeten dabei kognitive psychologische Techni- 
ken, um verschiedene Abweichungen vom «vernünftigen Verhalten» bei wirtschaftlichen Entscheidungen zu erklären.

Inzwischen wurden darüber hinaus vielfältige psychologische, genetisch- und kulturell-evolutorische Aspekte auf ihren Erklärungsgehalt für menschliches Verhalten überprüft (Fehr \& Fischbacher 2003). Es zeigte sich, dass Menschen in vielen Situationen soziale Präferenzen zeigen, d.h., sie handeln nicht allein aus Selbstinteresse, sondern beziehen das Wohlergehen beziehungsweise den Schaden anderer Akteure bei ihren Entscheidungen mit ein. Fehr \& Fischbacher (2002) gehen davon aus, dass ohne die Berücksichtigung dieser sozialen Präferenzen ein adäquates Verständnis grundlegender ökonomischer Zusammenhänge unmöglich ist. Dazu gehören unter anderem der Effekt von Wettbewerb auf das Marktergebnis, Regeln, die Kooperation und kollektives Handeln steuern sowie die Frage, welche Arrangements für Verträge und Eigentumsrechte optimal sind.

Die Theorien der Verhaltensökonomie werden häufig durch experimentelle Beobachtungen und Antworten auf Befragungen entwickelt beziehungsweise überprüft. Wissenschaftlich basierte Experimente, die Marktsituationen wie Börsenhandel und Auktionen simulieren, werden als besonders nützlich angesehen, um das beobachtete Marktverhalten auf verschiedene Weise zu erklären und die Zahl nachvollziehbarer Erklärungen einzugrenzen. Gleichzeitig kommen aber auch, beispielsweise im Rahmen der Neuroökonomik, Erkenntnisse der Gehirnforschung zur Anwendung.

Für die Analyse forstlicher Märkte in der Schweiz erscheint besonders das Verhalten auf der Anbieterseite interessant und erklärungsbedürftig. Ausgehend vom Geringfügigkeitsproblem der kleinen privaten Waldeigentümer (Pudack 2006) und vom hohen Anteil kommunaler Forstbetriebe und deren unterschiedlicher Rahmenbedingungen stellt sich die Frage, ob die Verhaltensökonomie hilfreiche Ansätze für die Erklärung und potenzielle Änderung des Anbieterverhaltens liefern kann. Insbesondere könnten Voraussetzungen, Möglichkeiten und Grenzen von Kooperationen zur gemeinsamen Holzvermarktung analysiert werden (vgl. Fischbacher \& Gächter 2010).

Urs Fischbacher geht in seinem Beitrag davon aus, dass sich die Waldwirtschaft durch drei fundamentale Eigenschaften auszeichnet: 1) die Langfristigkeit forstlicher Produktionsentscheidungen, 2) die Produktion vielfältiger öffentlicher Güter des Waldes $^{1}$ und 3) die ausgeprägte staatliche Regulierung (Fischbacher 2012, dieses Heft). Vor diesem Hintergrund ist zu prüfen, ob und wie sich die Holzmarktakteure von Akteuren anderer Wirtschaftszweige unterscheiden, beispielsweise in Bezug auf zeitliche oder soziale Präferenzen oder auf das Risi- koverhalten. Hier können experimentelle Befragungen wichtige Erkenntnisse und Erklärungen für ein bestimmtes Marktverhalten liefern.

\section{Grundlagen der agentenbasierten Marktmodellierung und ihrer Anwendung}

Die agentenbasierte Modellierung ist eine Methode zur Simulation von eigenständig handelnden und interagierenden Agenten mit dem Ziel, die Auswirkungen dieser Handlungen und Interaktionen auf einer übergeordneten Ebene, beispielsweise einem Markt, zu untersuchen. ABM gehört daher zu den Methoden der Mikrosimulation und folgt einem Bottom-up-Ansatz. Ergeben sich dabei auf höherer Ebene Auswirkungen, die nicht direkt aus dem Verhalten der Agenten auf Mikroebene ableitbar sind, so spricht man von emergentem Systemverhalten.

Eine Teildisziplin der agentenbasierten Modellierung, die sich speziell mit der ökonomischen Modellierung beschäftigt, wird als Agent-based Computational Economics (ACE) bezeichnet (Axelrod \& Tesfatsion 2006, Tesfatsion 2006). Die zentrale Frage dieser Forschungsrichtung ist, wie die Interaktionen von Individuen zu bestimmten ökonomischen Phänomenen führen. Dabei können auch die Erklärungsansätze ökonometrischer Modelle und Erkenntnisse der experimentellen Ökonomik genutzt werden. Aufgrund der Möglichkeiten, die dieser Ansatz bietet - die Simulation der Interaktion von Agenten mit heterogenem Verhalten in einem räumlichen Kontext und einer fast beliebig gestaltbaren Umwelt -, scheint ACE ideal geeignet zu sein, um Erkenntnisfortschritte in ökonomischen Fragestellungen zu erzielen, die durch klassische ökonometrische Modelle nur eingeschränkt analysierbar scheinen.

Die Agenten können sowohl Personen repräsentieren als auch soziale Gruppierungen und Institutionen. Sie können beispielsweise aber auch biologische Einheiten wie Wälder und physische Einheiten wie geografische Regionen darstellen. Möglich ist auch eine hierarchische Struktur, bei der Agenten aus verschiedenen anderen Agenten bestehen. Das Spektrum reicht von passiven Objekten ohne kognitive Funktion bis hin zu aktiven Entscheidungsträgern mit Lernkapazitäten. Die Agenten weisen Eigenschaften auf und agieren aufgrund dieser Eigenschaften auf unterschiedliche Weise miteinander. Bei den Eigenschaften kann es sich zum Beispiel um Typenattribute wie Holzanbieter und -nachfrager oder um Strukturattribute wie Hiebsätze und Holzerntekosten handeln. Bei den Interaktionen der

\footnotetext{
1 Siehe dazu die Schwerpunktnummer «Externalitäten von Wald und Waldwirtschaft» (Ausgabe 11/2011) der Schweizerischen Zeitschrift für Forstwesen.
} 
Agenten können sowohl «harte» Faktoren, wie rationales wirtschaftliches Verhalten, als auch «weiche», wie menschliches Verhalten in Form von Fairness und Loyalität, berücksichtigt werden. Obwohl einige Autoren agentenbasierte Modelle als sehr wichtig und vielversprechend betrachten, um die ökonomische Realität abzubilden und verstehen zu können (Farmer \& Foley 2009), kam die Methode in der Forstwirtschaft bisher nur vereinzelt zur Anwendung. Erste Ansätze in der Schweiz dienten dazu, regionale Stoffflüsse von Holz zu untersuchen (Binder et al 2003). Bezüglich der Analyse forstlicher Märkte in der Schweiz interessiert vor allem, ob und wie im Rahmen des Bottom-up-Ansatzes vom individuellen Verhalten der Waldeigentümer beziehungsweise der Förster auf ein Systemverhalten auf höherer Ebene geschlossen und beispielsweise ein bestimmtes Angebotsverhalten oder Marktergebnis erklärt werden kann. Man erhofft sich insbesondere Aufschluss über die tatsächliche Verfügbarkeit und Allokation des Waldholzes.

Klaus G. Troitzsch schlägt in seinem Beitrag vor, bestimmte Klassen von Holzmarktakteuren zu definieren und diese dann auf Basis von empirisch ermittelten Verhaltensweisen mit entsprechenden Merkmalen auszustatten. Durch eine sogenannte «deklarative Modellierung» können dann mögliche zukünftige Marktzustände und Konflikte aufgezeigt werden (Troitzsch 2012, dieses Heft).

Fabian Kostadinov und Kollegen stellen beispielhaft ein erstes agentenbasiertes Modell für einen Schweizerischen Energieholzmarkt vor. Sie schildern dabei die einzelnen Phasen der Entwicklung und verdeutlichen, welche Annahmen getroffen werden und welche Informationslücken gefüllt werden müs-

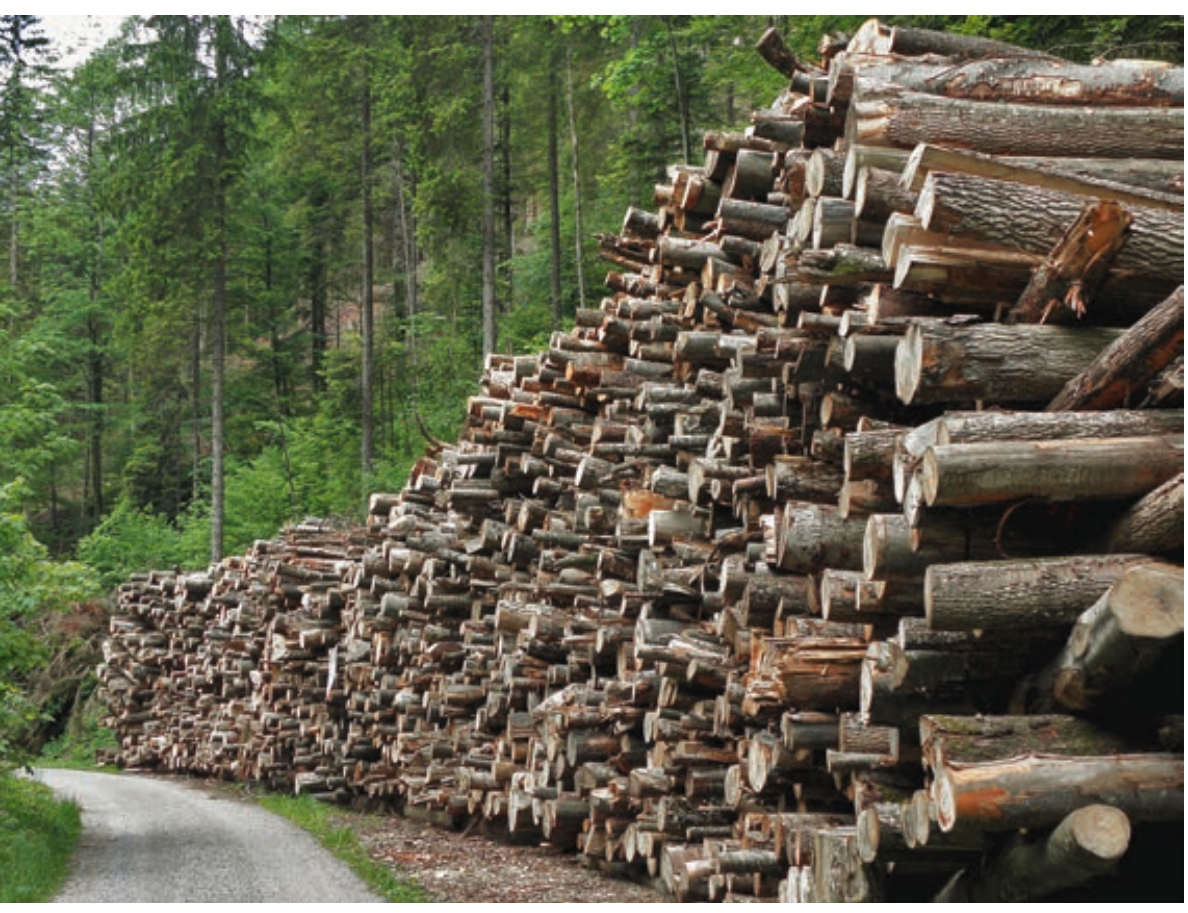

Abb 2 Der Energieholzmarkt ist ein weiterer Holzmarkt. In diesem sind langfristige Verträge üblich. Foto: Barbara Allgaier Leuch sen, um das Modell zu verbessern und realitätsnäher zu gestalten (Kostadinov et al 2012, dieses Heft).

\section{Die Funktionsweise von Holzmärkten aus der Sicht der Praxis}

Die theoretischen Betrachtungen zu den Holzmarktanalysen und ihren Ergebnissen wurden während des 8. Waldökonomischen Seminars durch Erfahrungsberichte aus der Praxis ergänzt. Es kamen Akteure zu Wort, die die Holzmärkte durch langjährige Tätigkeit als Verkäufer oder Käufer kennen (Abbildung 2). Auf der Angebotsseite waren Holzvermarktungszentralen des kleinteiligen öffentlichen und privaten Waldbesitzes und auf der Nachfrageseite grössere Holzabnehmer der stofflichen und energetischen Verwertung vertreten. Die Praxisberichte zeigten eindrücklich, wie vielschichtig die Grundlagen für Kauf- und Verkaufsentscheidungen in der Praxis sein können und dass von Fall zu Fall ganz unterschiedliche Rahmenbedingungen zu beachten sind.

\section{Schlussfolgerungen}

Das 8. Waldökonomische Seminar bot ein Forum, um neueste Erkenntnisse auf den Gebieten der empirischen, verhaltensökonomischen Marktanalyse sowie der agentenbasierten Modellierung zu präsentieren und zu diskutieren. Durch den Austausch zwischen Wissenschaft und Praxis konnten wertvolle Hinweise gewonnen werden, wie die verschiedenen Ansätze zur Beschreibung und Erklärung des Marktgeschehens in Zukunft weiterentwickelt werden können. In der Schweiz liefert das Nationale Forschungsprogramm NFP 66 «Ressource Holz» einen idealen Rahmen dafür. Konkret wurde ein Forschungsprojekt in Auftrag gegeben, in dem die oben angesprochenen Ansätze verknüpft werden. So soll geprüft werden, wie das Marktverhalten umfassend erklärt werden kann und welche Auswirkungen forstökonomische und forstpolitische Instrumente auf die Holzverfügbarkeit in der Schweiz haben können.

Eingereicht: 22. August 2012, akzeptiert (ohne Review): 24. August 2012

\section{Literatur}

ARMSTRONG M, PORTER R (2007) Handbook of industrial organization III. Amsterdam: Elsevier. 879 p.

AXELROD R, TESFATSION L (2006) A guide for newcomers to agent-based modeling in the social sciences. In: Tesfatsion $L$, Judd KL, editors. Handbook of computational economics, vol II: Agent-based computational economics. Amsterdam: Elsevier. pp. 1647-1659.

BERGEN V (2012) Grundlagen der empirischen Marktanalyse, dargestellt am Beispiel eines Rohholzmarktes. Schweiz Z Forstwes 163: 401-407. doi:10.3188/szf.2012.0401 
BERGEN V, LÖWENSTEIN W, OLSCHEWSKI R (2002) Forstökonomie - Volkswirtschaftliche Grundlagen. München: Vahlen. 469 p.

BINDER CR, HOFER C, WIEK A, SCHOLZ RW (2003) Transition process towards improved regional wood flow by integrating material flux analysis and agent analysis: the case of Appenzell Ausserrhoden, Switzerland. Zürich: ETH Zürich, Umweltnatur- und Umweltsozialwissenschaften. doi: 10.3929/ethza-004570841

FARMER JD, FOLEY D (2009) The economy needs agent-based modelling. Nature 460: 685-686.

FEHR E, FISCHBACHER U (2002) Why social preferences matter the impact of non-selfish motives on competition, cooperation and incentives. Econ J 112: C1-C33.

FEHR E, FISCHBACHER U (2003) The nature of human altruism. Nature 425: 785-791.

FISCHBACHER U (2012) Experimente und forstökonomische Fragen. Schweiz Z Forstwes 163: 417-421. doi: 10.3188/ szf.2012.0417

FISCHBACHER U, GÄCHTER S (2010) Social preferences, beliefs, and the dynamics of free riding in public good experiments. Am Econ Rev 100: 541-556.

KAHNEMAN D, TVERSKY A (1979) Prospect theory: An analysis of decisions under risk. Econometrica 47: 313-327.

KOSTADINOV F, STEUBING B, HOLM S (2012) Vorgehen zur agentenbasierten Modellierung eines schweizerischen Waldenergieholzmarktes. Schweiz Z Forstwes 163: 422-431. doi: 10.3188/szf.2012.0422

MANKIW NG, TAYLOR MP (2008) Grundzüge der Volkswirtschaftslehre. Stuttgart: Schäffer-Poeschel, 4 ed. 988 p.

MICHELS L (1991) Zum Angebotsverhalten privater Forstbetriebe. Forst Holz 46: 473-476.

MICHELS L, STEINMEYER U (1992) Zum Angebotsverhalten der Niedersächsischen Landesforstverwaltung auf dem Nadelstammholzmarkt von 1976 bis 1989. Forst Holz 47: 748-751.

MILLER AC (2003) Erwartungsbildung ökonomischer Akteure. Wiesbaden: Deutscher Universitätsverlag. 360 p.

MOOG M (1992) Zum Angebotsverhalten von Forstbetrieben: eine ökonometrische Studie. Frankfurt a.M.: Sauerländer. 298 p.
OLSCHEWSKI R, STEUBING B, LEMM R, THEES O (2009) Ansätze für eine agentenbasierte Modellierung von Holzmärkten. In: Thees $\mathrm{O}$, Lemm R, editors. Management zukunftsfähige Waldnutzung. Zürich: VDF. pp. 225-244.

PAULI B, BÜRGI P, BRÜLHART S (2010) Holz als Rohstoff und Energieträger. Dynamisches Holzmarktmodell und Zukunftsszenarien. Schlussbericht zu Handen BFE und BAFU. www.bafu. admin.ch/aktionsplan-holz/10302/index.html?lang=de (29.8.2012)

PUDACK T (2006) Ansatzpunkte für den Strukturwandel in der Forstwirtschaft. Schweiz Z Forstwes 157: 73-81. doi: 10.3188/ szf. 2006.0073

SCHWARZBAUER P, STERN T (2010) Energy vs. material: Economic impacts of a "wood-for-energy scenario" on the forest-based sector in Austria - a simulation approach. For Pol Econ 12: $31-$ 38.

SHOGREN JF, PARKHURST GM, BANERJEE P (2010) Two cheers and a qualm for behavioral environmental economics. Environ Res Econ 46: 235-247.

STEINMEYER U (1992A) Der deutsche Nadelstammholzmarkt: eine aktualisierte Modellschätzung und Untersuchungen zu einem besitzartenspezifischen Angebotsverhalten von Forstbetrieben. Forstarchiv 63: 106-111.

STEINMEYER U (1992B) Der bundesdeutsche Industrieholzmarkt von 1965 bis 1987. Frankfurt a.M.: Sauerländer. 267 p.

TESFATSION L (2006) Agent-based computational economics: a constructive approach to economic theory. In: Tesfatsion L, Judd KL, editors. Handbook of computational economics, vol II: Agent-based computational economics. Amsterdam: Elsevier. pp. 831-880.

THOROE C, SASSE V, ENGLERT H, ELSASSER P (1998) Zum potentiellen Aufkommen und Angebot von Rohholz: Inverses Angebotsverhalten auf deutschen Märkten nicht bestätigt. HolzZent.bl 124: 577-584.

TROITZSCH KG (2012) Agentenbasierte Modellierung von Märkten. Schweiz Z Forstwes 163: 408-416. doi: 10.3188/ szf.2012.0408

\section{Möglichkeiten und Grenzen der Analyse von Holzmärkten}

Neuere Ansätze der Verhaltensökonomie und der agentenbasierten Modellierung (ABM) können das Verständnis des Marktgeschehens und die Beurteilung zukünftiger Entwicklungen im Vergleich zu den traditionellen ökonometrischen Methoden erweitern. Dies vor allem durch Fokussierung auf das individuelle Verhalten der Akteure und Szenarioanalysen. Der Beitrag fasst zusammen, was die Analysemethoden leisten können, um die Realität der Märkte besser zu beschreiben, zu erklären und vorherzusagen. Die Ausführungen basieren auf dem aktuellen Stand des Wissens und reflektieren die Erkenntnisse des 8. Waldökonomischen Seminars, das im Herbst 2011 stattfand und bei dem neben Wissenschaftlern auch Praktiker ihre Erkenntnisse präsentierten.

\section{Possibilités et limites de I'analyse des marchés du bois}

Les nouvelles approches de l'économie comportementale et de la simulation à base d'agents peuvent améliorer la compréhension des processus du marché et l'évaluation des développements futurs en mettant l'accent sur le comportement individuel des acteurs du marché et sur l'analyse des scénarios. Dans cet article, nous résumons les possibilités de cette approche en termes de description, de compréhension et pronostic des développements réels du marché. L'exposé se base sur l'état des connaissances actuel et reflète les conclusions du 8 e séminaire d'économie forestière qui a permis, en automne 2011, aux scientifiques et aux praticiens de présenter leurs résultats. 